\title{
Nanoscale charging hysteresis measurement by multifrequency electrostatic force spectroscopy
}

\author{
Umut Bostanci, ${ }^{1, a)}$ M. Kurtuluş Abak, ${ }^{2}$ O. Aktaş, ${ }^{2}$ and A. Dâna ${ }^{2, b)}$ \\ ${ }^{1}$ Department of Physics, Middle East Technical University, 06531 Ankara, Turkey \\ ${ }^{2}$ Institute of Materials Science and Nanotechnology, Bilkent University, 06800 Ankara, Turkey
}

(Received 2 November 2007; accepted 7 February 2008; published online 4 March 2008)

\begin{abstract}
We report a scanning probe technique that can be used to measure charging of localized states on conducting or partially insulating substrates at room temperature under ambient conditions. Electrostatic interactions in the presence of a charged particle between the tip and the sample is monitored by the second order flexural mode, while the fundamental mode is used for stabilizing the tip-sample separation. Cycling the bias voltage between two limits, it is possible to observe hysteresis of the second order mode amplitude due to charging. Results are presented on silicon nitride films containing silicon nanocrystals. (C) 2008 American Institute of Physics.
\end{abstract}

[DOI: $10.1063 / 1.2888765$ ]

Atomic force microscopy $(\mathrm{AFM})^{1}$ is a powerful tool for imaging various tip-sample interactions on the nanometer scale. Imaging electronic properties are especially important for nanoelectronics; and electrostatic force microscopy has been applied to, for example, electrical characterization of carbon nanotubes, ${ }^{2}$ DNA, ${ }^{3}$ and nanoparticles. ${ }^{4}$ Kelvin probe microscopy, which measures the contact potential difference between the tip and the sample, was applied to the label-free detection of nucleotides. ${ }^{5}$ Electrostatic force microscopy has been used to study light induced carrier dynamics in organic conductors. ${ }^{6}$ Single carrier charging events have been observed by AFM. ${ }^{7,8}$ Recently, multifrequency imaging has been applied to imaging of electrostatic forces on insulating substrates. ${ }^{9}$

In this letter, we report an extension of multifrequency AFM to a spectroscopy technique similar to capacitance spectroscopy. We refer to the technique as the multifrequency electrostatic force spectroscopy (MFEFS). The technique is demonstrated by observing charging hysteresis of silicon nanocrystals in a silicon nitride matrix on silicon. In the experiments, a commercial AFM tip was used. The tip was a Pt/Ir coated rectangular cantilever with a nominal length of $L=220 \mu \mathrm{m}$, width of $w=30 \mu \mathrm{m}$, tip height of $H$ $=15 \mu \mathrm{m}$, tip half-cone angle of $\theta=15^{\circ}$, and nominal tip radius of $R=15 \mathrm{~nm}$. The fundamental mode of the cantilever is used to stabilize the average tip-sample separation, and the second order mode is used to resonantly measure electrostatic forces. In order to limit the charge transfer between the biased tip and the surface, and to avoid instabilities during tapping, the tip is coated with a nominal $t_{d}=10 \mathrm{~nm}$ thick $\mathrm{Si}_{3} \mathrm{~N}_{4}$ layer in a plasma-enhanced chemical-vapor deposition (PECVD) reactor (PlasmaLab 8510C) using $\mathrm{SiH}_{4}$ and $\mathrm{NH}_{3}$ as precursor gases. This layer is analogous to the gate oxide in flash memory devices, and the tip can be considered as a metallic gate. The first and second eigenfrequencies of the cantilever were measured to be $f_{0} \cong 70.01 \mathrm{kHz}$ and $f_{2}$ $\cong 448.83 \mathrm{kHz}$, with quality factors of $Q_{0}=220$ and $Q_{2}=450$. The experiments were performed on a commercial AFM system (PSIA XE100E) under ambient conditions. During imaging, the cantilever is driven at a frequency slightly below

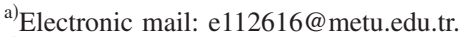

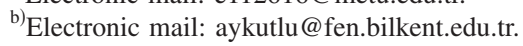

$f_{0}$ by the dither piezo, and a sinusoidal voltage superimposed on a dc voltage is applied at a frequency that coincides with the second order flexural mode frequency $\omega_{2}=2 \pi f_{2}$. The deflection of the second-order mode is measured using an external rf lock-in amplifier (Stanford Research Systems SR844). It is known that, unless the second order mode frequency is an integer multiple of the fundamental mode frequency, tapping at the fundamental frequency does not generate significant oscillation of the second order mode. ${ }^{10,11}$ Using this fact and separate excitation sources for the two modes, it is possible to separate short and long range interactions by measuring the oscillation amplitudes and phase at $f_{0}$ and $f_{2}$. In order to minimize the coupling of the two modes to each other through nonlinearities imposed by the tip-sample interaction, the second order mode amplitude $A_{2}$ was kept below $1 \mathrm{~nm}$. Topographic feedback is maintained by the feedback loop of the AFM system using the fundamental mode. Typical fundamental mode amplitudes were $A_{0} \simeq 10-20 \mathrm{~nm}$ defined by the adjustable set point. The tipsample interaction force can be written as the sum of electrostatic and nonelectrostatic terms as $F_{\mathrm{ts}}=F_{e}+F_{\text {ne. }}$ The electrostatic component is dependent on the tip-sample bias $V_{\mathrm{ts}}$. In our experiments, the tip-sample voltage $V_{\mathrm{ts}}$ is of the form $V_{\mathrm{ts}}(t)=V_{\mathrm{dc}}+V_{\mathrm{ac}} \cos \left(\omega_{2} t\right), \quad V_{\mathrm{ac}}$ typically being about 0.1 to $1 \mathrm{~V}$. This modulated bias results in an average electrostatic force amplitude $F_{e, \omega_{2}}$ at frequency $f_{2}$ that can be written as

$$
F_{e, \omega_{2}}=\left\langle\frac{\partial C_{t s}(z)}{\partial z}\right\rangle\left(V_{\mathrm{dc}}-V_{\mathrm{cpd}}\right) V_{\mathrm{ac}}
$$

where $C_{\mathrm{ts}}(z)$ is the tip-sample capacitance, $V_{\mathrm{dc}}$ is the tipsample bias, and $V_{\mathrm{CPD}}$ is the contact potential difference. Brackets denote averaging over the oscillation period. When the ac excitation amplitude $V_{\mathrm{ac}}$ is kept constant, $F_{e, \omega_{2}}$ is linearly proportional to $V_{\mathrm{dc}}-V_{\mathrm{CPD}}$.

In order to verify that the second order mode oscillation is excited primarily by electrostatic interactions, we sweep the dc bias or the frequency of $V_{\mathrm{ac}}$ and record the second order mode amplitude and phase, while the first order mode is tapping a flat and clean silicon surface. The excitation amplitude of the second order mode follows the dc voltage bias, and is nulled when the contact potential difference is 


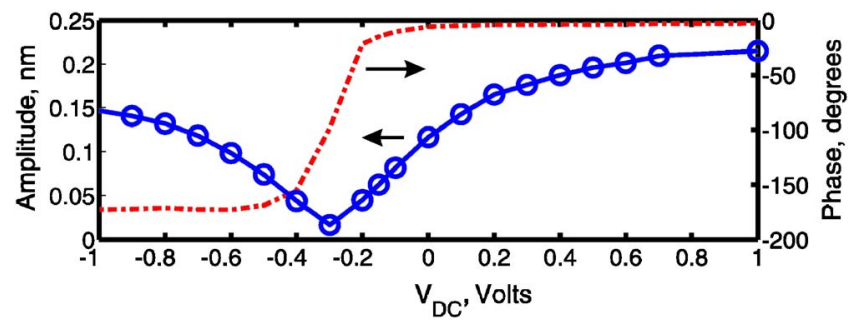

FIG. 1. (Color online) Dependence of the amplitude of the second order mechanical mode on dc bias voltage, during tapping with the fundamental mode. The ac frequency was on resonance with the second order mode. Smooth transition of the phase of the oscillation indicates finite capacitive coupling of the drive signal to the dither piezo.

compensated around $-0.3 \mathrm{~V}$, as seen in Fig. 1, in accordance with Eq. (1). For large biases, detachment of the tip from the sample surface takes place. This effect is previously well studied and can be understood by considering the coupling of the dc electrostatic forces to the topography channel. ${ }^{12}$ Tip retraction results in a reduced average electrostatic excitation of the second order mode, due to the increased average tipsample separation. The feedback effect explains the observed deviation from linearity in the excitation amplitude at bias differences greater than $0.5 \mathrm{~V}$ shown in Fig. 1. This effect must be avoided or be carefully considered when quantitative measurements under large biases are needed.

If a localized state is separated from the bulk of the substrate by a tunnel barrier, applying an electric field large enough to make the tunnel barrier transparent for carrier transport will result in accumulated charge in the localized state. When the field is reduced, the carriers will stay in the localized state until they slowly tunnel out, or a large enough reverse polarity field causes them to escape. In order to interpret the data collected during a measurement of localized charging, the electrostatic model has to include the effect of localized charges. For the sake of simplicity, we consider the case of a nanocrystal below the tip. The electrostatic interaction energy between the tip and the sample with a charge trapped in a nanocrystal in between is given by ${ }^{13,14}$

$$
U=\frac{q^{2}}{2 C_{t}}-\frac{C_{1}}{2 C_{t}} q V-\frac{C_{1} C_{2}}{2 C_{t}} V^{2}-\frac{C_{0}}{2} V^{2},
$$

where $C_{0}$ is the tip-substrate capacitance, $C_{1}$ is the tipnanocrystal capacitance, $C_{2}$ is the nanocrystal-substrate ca-

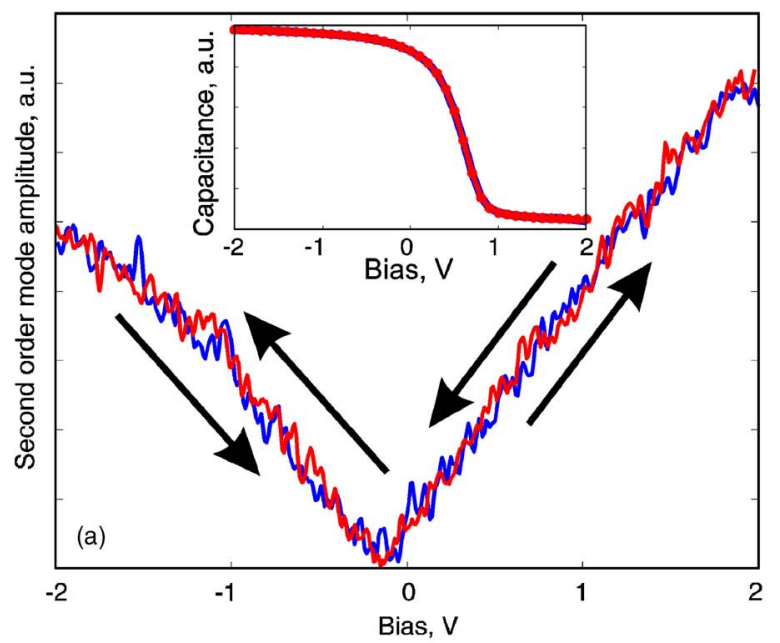

pacitance, and $C_{t}=C_{1}+C_{2}$ total capacitance seen by the tip. The electrostatic force can be obtained from Eq. (2) by differentiation with respect to the tip-sample separation $z$,

$$
F_{e}=\frac{1}{C_{t}} \frac{\partial C_{1}}{\partial z}\left(\frac{q^{2}}{2}-C_{2} q V+\frac{C_{2}^{2} V^{2}}{2}\right)+\frac{1}{2} \frac{\partial C_{0}}{\partial z} V^{2} .
$$

Using Eq. (3), and the ac modulated bias, different frequency components of the electrostatic force can be evaluated. When the electrostatic excitation is measured as a function of the bias, the presence of charge in a state between the tip and the sample results in a shift of the curve along the bias coordinate. Using algebraic manipulation, Eq. (3) can be used to calculate the hysteresis between the force-voltage curves with a charge $q$ deposited in between the two curves, given by

$$
\Delta V_{H}=\frac{q}{C_{2}}\left[1+\frac{\partial C_{0}}{\partial z} /\left(\frac{C_{2}}{C_{1}+C_{2}}\right)^{2} \frac{\partial C_{1}}{\partial z}\right]^{-1} .
$$

The voltage $\Delta V_{H}$ is the charging voltage of the trap $V_{c}$ $=q / C_{2}$, divided by a dimensionless factor that quantifies the shielding effect of the tip. Using Eq. (4), by measuring the hysteresis at specific location, information about the local density of states and charging-discharging dynamics can be obtained. The technique was demonstrated on different sets of samples. Figure 2(a) shows the typical result for the amplitude of the second order mode during the cyclic voltage sweep on a clean silicon surface. No hysteresis can be observed, due to the low density of surface states on a clean silicon surface. Figure 2(b) shows the typical result of the same experiment performed on a $10 \mathrm{~nm}$ thick silicon nitride film with embedded silicon nanocrystals grown by PECVD (see supporting information). The average diameter of silicon nanocrystals is $5 \mathrm{~nm}$ inferred from photoluminescence measurements. Significant hysteresis of about $310 \mathrm{mV}$ can be observed. Assuming a nominal nanocrystal diameter of $5 \mathrm{~nm}$ and a nominal conducting tip radius of $15 \mathrm{~nm}$, through finite element analysis, the relevant capacitances and gradients are estimated as $C_{1}=1.05 \mathrm{aF}, C_{2}=1.4 \mathrm{aF}, \partial C_{0} / \partial z=120 \mathrm{pF} / \mathrm{m}$, and $\partial C_{1} / \partial z=25 \mathrm{pF} / \mathrm{m}$. These values give a hysteresis voltage of $56 \mathrm{mV}$ per electron per nanocrystal. The measured hysteresis of $310 \mathrm{mV}$ corresponds to about six electrons per nanocrystal, deposited between 0 and 2 V. Arrows in Fig.

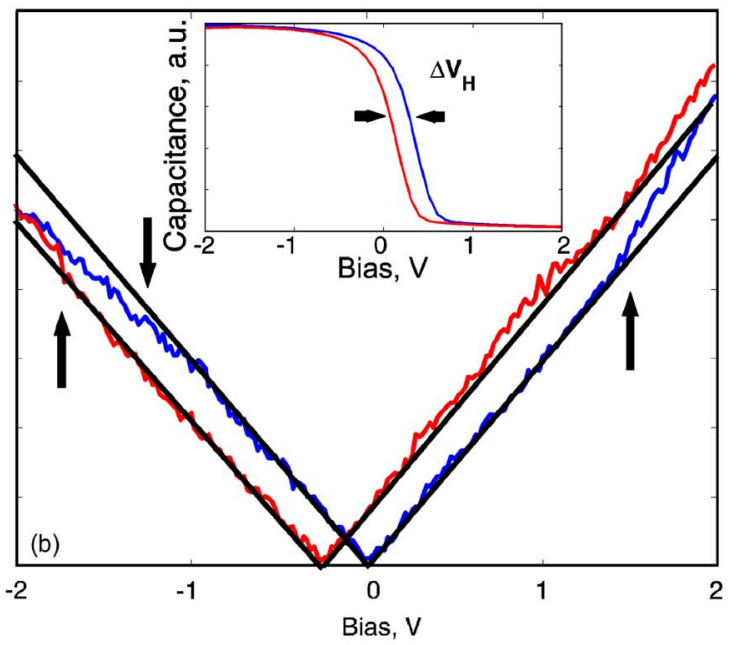

FIG. 2. (Color online) Electrostatic excitation of the second order mode during a voltage sweep on a clean silicon surface. The arrows denote voltage sweep directions. No hysteresis can be observed. (b) Electrostatic excitation of the second order mode during a voltage sweep on silicon nitride layer with silicon nanocrystals embedded. Significant hysteresis is observable, indicating charging of nanocrystals. The arrows denote onset of charging and discharging events. 


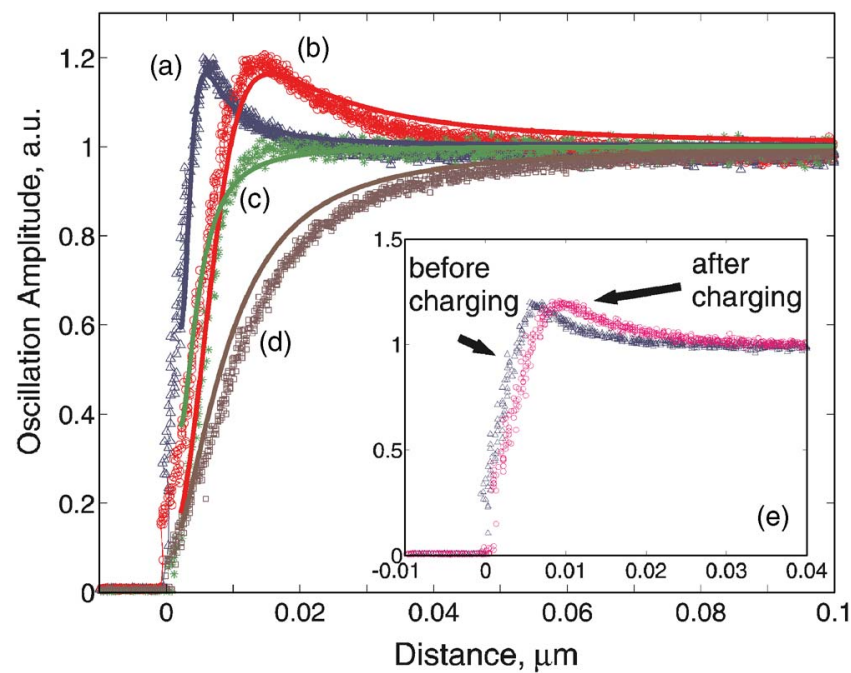

FIG. 3. (Color online) Oscillation amplitude vs tip-sample separation. When the cantilever is driven below its resonance frequency, peaking of the oscillation amplitude is observed (a) for $0 \mathrm{~V}$ bias and (b) for $2 \mathrm{~V}$ bias. If the drive frequency is above the resonance frequency, no such peaking occurs as seen in (c) for $0 \mathrm{~V}$ bias and (d) for $2 \mathrm{~V}$ bias. Representative theoretical fits obtained by using the same set of parameters and by only changing the bias parameter are shown as solid curves. (e) The inset shows amplitude vs tip-sample separation scans obtained at $0 \mathrm{~V}$ bias before and after charging the film by contacting with a $2 \mathrm{~V}$ biased tip. Shift of the curve and broadening of the peak after charging indicate a local surface potential shift.

2(b) denote onset of charging and discharging of the nanocrystals. For our signal to noise ratio, single electron events should be observable for nanoparticles with up to $10 \mathrm{~nm}$ diameter, $3 \mathrm{~nm}$ away from the substrate, and using a $15 \mathrm{~nm}$ radius tip. The exact hysteresis value for a single electron depends on other parameters of the sample such as the thickness and dielectric constant of the tunnel barrier. In order to highlight the analogy of MFEFS with conventional capacitance spectroscopy, macroscopic capacitors $(1 \mathrm{~mm}$ gate diameter) were fabricated on nonstoichiometric silicon nitride films containing nanocrystals and on stoichiometric silicon nitride films without nanocrystals. The capacitance-voltage traces shown in the insets of Figs. 2(a) and 2(b) are acquired using an $L C R$ meter and display similar hysteresis.

In order to ensure that the observed effects are due to charging and not due to instability caused by tip-sample interactions, it is necessary to investigate the tip-sample interactions in greater detail. Figure 3 shows amplitude-distance data obtained at different biases. The inset of Fig. 3 shows that long-range electrostatic interactions are indeed perturbed as a result of charging of the film. Amplitude distance curves obtained at zero bias differ before and after charging. The effect of charging is roughly equivalent to applying a bias $\Delta V_{H}$ to the cantilever. The observations are well understood by considering the cantilever dynamics taking into account the electrostatic and nonelectrostatic interactions. Numerical calculations of how the oscillation amplitude of the fundamental resonant mode changes with bias and tip-sample separation is shown as solid curves in Fig. 3. Measurement results and simulation results of the tip-sample interaction indicate that, in the repulsive mode, the amplitude distance curves are single valued for tip-sample separations below the free oscillation amplitude. For drive frequencies below the zero-bias resonant frequency, peaking of the amplitude can be observed if the tip-sample separation is greater than the free oscillation amplitude. For stable operation of MFEFS, this parameter range must be avoided and amplitude set point of topographic feedback must be chosen below 90 percent of free oscillation amplitude. For drive frequencies above zerobias resonant frequency, there is no peaking that may potentially result in bistabilities. However, in this parameter range, coupling of the electrostatic interactions to the topography feedback are more pronounced. There are advantages of the MFEFS technique to force or amplitude versus distance measurements. In MFEFS, the tip is never brought into hard contact with the sample, therefore both the sample and the tip structures are preserved. In conventional force versus distance measurements, instrument drifts are not independently compensated; however, MFEFS inherently stabilizes the tipsample separation. Bias sweeps in a conventional force versus distance measurement requires complicated data analysis, whereas MFEFS data directly give a hysteresis voltage that can be related to charging through characterization of the tip shape. Ideally, MFEFS measurements can be done rapidly (about $Q_{2} / f_{2} \approx 1 \mathrm{~ms}$ per bias point), therefore, spatial drifts of instrumentation can have less effect on measurements.

The MFEFS technique we presented here can be applied using a standard AFM system using a standard metal coated cantilever in ambient conditions. For conductive substrates and samples without a cap layer, a thin barrier coating on the tip can be applied to improve stability. The technique can be regarded as a technique that is similar to scanning tunneling spectroscopy (STS) used in scanning tunneling microscopy studies. However, it can be used to study trap levels on partially insulating samples where STS can not be applied. Detailed description of excitation of the second order mode by ac electrostatic force; Finite element calculation results of tip-nanocrystal and nanocrystals substrate capacitances; Preparation of silicon nitride films containing silicon nanoparticles can be found in the supporting information.

This work has been partially funded by Tübitak Grant 104T520 and 107T547, and by funding from the State Planning Agency of the Turkish Republic. Aykutlu Dâna thanks Atilla Aydinli for letting use of the capacitance measurement setup.

${ }^{1}$ G. Binnig, C. F. Quate, and C. Gerber, Phys. Rev. Lett. 56, 930 (1986).

${ }^{2}$ T. S. Jespersen and J. Nygard, Nano Lett. 5, 1838 (2005).

${ }^{3}$ M. Bockrath, N. Markovic, A. Shepard, and M. Tinkham, Nano Lett. 2, 187 (2002).

${ }^{4}$ R. M. Nyffenegger, R. M. Penner, and R. Schierle, Appl. Phys. Lett. 71, 13 (1997).

${ }^{5}$ A. K. Sinensky and A. M. Belcher, Nat. Nanotechnol. 2, 653 (2007).

${ }^{6}$ D. C. Coffey and D. S. Ginger, Nat. Mater. 5, 735 (2006).

${ }^{7}$ A. Dâna and Y. Yamamoto, Nanotechnology 16, S125 (2005).

${ }^{8}$ E. Bussmann and C. C. Williams, Appl. Phys. Lett. 88, 263108 (2006).

${ }^{9}$ R. W. Stark, N. Naujoks, and A. Stemmer, Nanotechnology 18, 065502 (2007).

${ }^{10}$ R. W. Stark and W. M. Heckl, Surf. Sci. 457, 219 (2000).

${ }^{11}$ O. Sahin, C. F. Quate, O. Solgaard, and A. Atalar, Phys. Rev. B 69, 165416 (2004)

${ }^{12}$ D. Ziegler, J. Reichen, N. Naujoks, and A. Stemmer, Nanotechnology 18, 225505 (2007).

${ }^{13}$ D. M. Schaadt, E. T. Yu, S. Sankar, and A. E. Berkowitz, Appl. Phys. Lett. 74, 472 (1999).

${ }^{14}$ Y. Azuma, M. Kanehara, T. Teranishi, and Y. Majima, Phys. Rev. Lett. 96, 016108 (2006). 